Cardiology 1996;87:573-574

\title{
Subject Index Vol. 87,1996
}

\section{ACE inhibitor 409}

$\alpha$-Actinin 283

Acute aortic regurgitation 276

chest pain 331

myocardial infarction $67,257,331,354,361,415,529$

- $\quad$ - -, anterior wall 125

Adenosine 216, 516

Adverse events 409

Age 396,529 Ambulatory blood pressure monitoring 374

- $\quad$ monitoring 104

American Medial Association

Classification 443

- $\quad$ Society of Echocardiog-

raphy 429

Anaerobic threshold 147

Angina pectoris $12,60,86,287$

Angioplasty 396

Angiotensin-converting enzyme inhibitor 54

Antiarrhythmic drugs 33

Antiglobulin consumption test 67

Anti-heart mitochondrial antibodies 67

Antihypertensive therapy 409

Aortic stenosis 545

- $\quad$ valve 212

Apical hypertrophic cardiomy-

opathy 488 Arrhythmias 300 Arterial compliance 415 Arteries 415 Atenolol 402 Atherosclerosis

384,453 Atrial arrhythmias 181

fibrillation 98, 205, 537

flutter and fibrillation 86

function 98, 205

natriuretic factor 46

- $\quad$ septal defect 358

Autonomic nervous system 28,

200 Autonomous nervous system 402

Beta-blocker 409,492 Bioprosthesis 212 Bland-White-Garland syndrome 358

Blood pressure 374

- $\quad$ - elevations 119

Bradyarrhythmias, propofol

319 Bradycardia 300 
Cachexia 476 Canadian Cardiovascular

Society Classification 443 Captopril 18,153 Cardiac arrhythmias 502

conduction system 319

troponinT 392 Cardiomyopathy, dilated 141,

177 Cardioplegia 516 Cardiopulmonary bypass 509

- $\quad$ stress testing 147

Cardioversion, direct current

181 Catheter 276

- $\quad$ ablation 42

Catheterization 82

cGMP 46

Chagas' cardiomyopathy 481 Chest pain 60

wall syndromes 60 Cholesterol 1,469

esterification 458 Cigarette smoking 502 Cilazapril 54, 402, 524 Circadian rhythm 374

Circulating intercellular adhesion molecule-1 189

Circulatory models 415 Clinical trials 453 Collateral circulation 358 Complement fixation test 67

Complete atrioventricular

block 71 Compliance abnormality 436 Complications 396 Congestive heart failure 46, 86, $147,153,250,325$ Contractile phenotype 23 Contrast, spontaneous 141 Coronary angioplasty 257 anomaly 260

arteriography 263,497

artery disease $60,134,244,306,343,453,492,497,560$

- fistulae 263

blood flow 306

disease 271

fistula 325

flow heterogeneity 294

heart disease 1, 552

hemodynamics 6

spasm 361

surgery 260

thrombus 384

vasodilator reserve 6 Counterpulsation 271 Creatine kinase 392 Cyclosporine 469

Diabetes 492

Diabetic neuropathy 283

Diastolic augmentation 537

- $\quad$ function 287

Dilated cardiomyopathy 502 Diphtheria 79 Disopyramide 6 Dobutamine 161

- $\quad$ stress echocardiography 82 ,

244

Doppler echocardiography 98 ,

205,212,545 Drainage pattern 263 Drug safety 409

Echocardiography 79, 169, 181, 250,279,287,429,566

-, transesophageal 112,141 
Ejection fraction 125, 250, 481

Elderly 429

Electrical cardioversion 98

Electrocardiogram 82, 125

Electrocardiography 79, 429

ELISA 189

Endocardium 23

Endothelin-1 303

Epidemiology 1,429

Essential hypertension 524

Exercise 343

Exercise stress test(ing) 147, 343

testing 134

tolerance 271

Familial hypercholesterolemia

469 Farnesol 458 Fibric acid analogues 469 Fibrinogen 524

Gastro-oesophageal disorder 60 Gender 230 Geranylgeraniol 458 Giant negative T wave(s) 365,

488

Heart block 76

catheterization 545

failure $12,358,481$

rate 28

- variability 104,119 ,

402,492

Hemodynamics, left atrial 112

Histocompatibility complexes 488

HMG-CoA-reductase inhibitors 469

Holier monitoring 300

Hydrochlorothiazide 313

Hyperlipidemia 469

Hypertension 54,313

Hypertrophic cardiomyopathy 436

- $\quad$ obstructive cardiomyopathy

6

Hypotension 374 Hypothermia 516

Impedance cardiography 147

- $\quad$ measurement 42

Interleukin 476

Intra-aortic balloon counterpul

sation 306

Intravascular ultrasonography

354 Inverted T wave 91 Isch(a)emic heart disease 161,

331 Ischemia 82, 343 Ischemic preconditioning 216 Isovolumic relaxation time 560

Klinefelter syndrome 358

Laser 384

Left atrial appendage 537 
- - flow 71

- pressure 224

coronary artery 260

ventricle 263

ventricular dysfunction 125

- filling 153

- function 347,415

573 